\title{
The Prognostic Impact of Synchronous Ipsilateral Multiple Breast Cancer: Survival Outcomes according to the Eighth American Joint Committee on Cancer Staging and Molecular Subtype
}

Jinah Chu $\cdot$ Hyunsik Bae Youjeong Seo · Soo Youn Cho Seok-Hyung Kim · Eun Yoon Cho

Department of Pathology and Translational Genomics, Samsung Medical Center, Sungkyunkwan University School of Medicine, Seoul, Korea

Received: July 31, 2018

Revised: September 27, 2018

Accepted: October 2, 2018

\section{Corresponding Author}

Eun Yoon Cho, MD, PhD

Department of Pathology and Translational

Genomics, Samsung Medical Center,

Sungkyunkwan University School of Medicine,

81 Irwon-ro, Gangnam-gu, Seoul 06351, Korea

Tel: +82-2-3410-2796

Fax: +82-2-3410-0025

E-mail: eunyoon.cho@samsung.com

Seok-Hyung Kim, PhD

Department of Pathology and Translational

Genomics, Samsung Medical Center,

Sungkyunkwan University School of Medicine,

81 Irwon-ro, Gangnam-gu, Seoul 06351, Korea

Tel: +82-2-3410-2898

Fax: +82-2-3410-0025

E-mail: parmenides.kim@samsung.com
Background: In the current American Joint Committee on Cancer staging system of breast cancer, only tumor size determines T-category regardless of whether the tumor is single or multiple. This study evaluated if tumor multiplicity has prognostic value and can be used to subclassify breast cancer. Methods: We included 5,758 patients with invasive breast cancer who underwent surgery at Samsung Medical Center, Seoul, Korea, from 1995 to 2012. Results: Patients were divided into two groups according to multiplicity (single, $n=4,744$; multiple, $n=1,014$ ). Statistically significant differences in lymph node involvement and lymphatic invasion were found between the two groups $(p<.001)$. Patients with multiple masses tended to have luminal A molecular subtype $(p<$ .001). On Kaplan-Meier survival analysis, patients with multiple masses had significantly poorer disease-free survival (DFS) $(p=.016)$. The prognostic significance of multiplicity was seen in patients with anatomic staging group $I$ and prognostic staging group $I A(p=.019$ and $p=.032$, respectively). When targeting patients with T1-2 NO M0, hormone receptor-positive, and human epidermal growth factor receptor 2 (HER2)-negative cancer, Kaplan-Meier survival analysis also revealed significantly reduced DFS with multiple cancer $(p=.031)$. The multivariate analysis indicated that multiplicity was independently correlated with worse DFS (hazard ratio, 1.23; 95\% confidence interval, 1.03 to $1.47 ; p=.025)$. The results of this study indicate that tumor multiplicity is frequently found in luminal A subtype, is associated with frequent lymph node metastasis, and is correlated with worse DFS. Conclusions: Tumor multiplicity has prognostic value and could be used to subclassify invasive breast cancer at early stages. Adjuvant chemotherapy would be necessary for multiple masses of T1-2 N0 M0, hormone-receptor-positive, and HER2-negative cancer.

Key Words: Breast neoplasms; Multiplicity; Disease-free survival; Prognosis; Molecular subtype
Several studies have been conducted on the multiplicity of breast cancer over the past decades. Multiplicity in breast cancer is a concept that includes multifocality and multicentricity. The term multifocality is used when there are two or more invasive tumors within the same breast quadrant, while the term multicentricity is used to denote the existence of tumors in different quadrants. ${ }^{1}$ The reported incidence of multiple breast cancers ranges from less than $10 \%$ to $70 \% .^{2-4}$ Multiplicity in breast cancer has been reported to correlate with a higher frequency of lymphovascular invasion and lymph node involvement. ${ }^{3,5-15}$ However, the clinical significance of multiplicity and its influence on prognosis are controversial. Therefore, the current edition of the tumor, node, and metastasis (TNM) classification in breast cancer uses only the dimension of the largest tumor focus when the tumor demonstrates multiplicity. In light of this fact, the present research analyzed, via a large retrospective study of breast cancer patients uniformly treated at a single institution, the impact of multiple breast cancers on disease-specific survival in relation to other clinicopathological factors.

\section{MATERIALS AND METHODS}

\section{Study population}

We identified 5,758 patients with invasive breast cancer who 
underwent conserving breast surgery or total mastectomy at Samsung Medical Center in Seoul, Korea, from 1995 to 2012. For inclusion in the study, patients needed to meet the following criteria: no distant metastasis at the time of diagnosis, no neoadjuvant therapy prior to surgery, and a follow-up period longer than 36 months. The mean age of the patients was 47 years (age range, 21 to 86 years), and the median follow-up period was 64 months. This study was approved by the Institutional Review Board (IRB) of Samsung Medical Center (IRB No. 2018-06098-001). Formal written informed consent was not required due to a waiver by the appropriate IRB.

\section{Clinicopathological evaluation}

Clinicopathological information, including multiplicity, age, tumor size, axillary nodal status, and histological grade, was obtained from electronic medical records or surgical pathology reports. According to the eighth edition of the American Joint Committee on Cancer (AJCC) staging, a patient with multiple breast cancer was defined if two or more separate masses were grossly or microscopically identified in a resection specimen no matter whether they were present in the same or different quadrants. In some cases, through assistance of careful gross examination and correlation with imaging findings, we can determine multiple breast cancer. Pathological tumor stage was assessed according to the eighth AJCC TNM classification. ${ }^{16}$ If an invasive carcinoma has been transected by vacuum-assisted biopsy or excisional biopsy, then the sizes in each fragment were not added together, and correlation with the size on breast imaging was helpful to determine the best size for classification. If there had been a prior core needle biopsy or incisional biopsy showing a larger area of invasion than in the excisional specimen, the largest dimension of the invasive carcinoma in the prior specimen should be used for $\mathrm{T}$ classification. Histological grade was evaluated according to the Scarff-Bloom-Richardson classification modified by Elston and Ellis. ${ }^{16}$ The expression status of estrogen receptors (ER), progesterone receptors (PR), and human epidermal growth factor receptor 2 (HER2) were evaluated by immunohistochemistry based on the surgical specimen. For ER and PR, only nuclear (not cytoplasmic) staining was scored. A positive test was defined as positive staining greater than or equal to $1 \%$ of tumor cells. A negative test was defined as staining of less than $1 \%$ of tumor cells. HER2 was scored as $0,1+, 2+$, or $3+$. Only membrane staining intensity and pattern were evaluated using the recommendations of the American Society of Clinical Oncology/College of American Pathologists. ${ }^{17,18} \mathrm{~A}$ positive test was defined as a staining score of $3+$. Tumors with a $2+$ score were submitted for silver in situ hybridization. The tumor was considered positive for HER2 amplification if the HER2/chromosome 17 probe signal ratio was greater than 2.0 and/or the average HER2 copy number was greater than 6.0 signals per cell. Molecular subtypes of breast cancer were classified into luminal A, luminal B1, luminal B2, HER2, and triple-negative subtypes based on histological grade and the results of ER, PR, and HER2 immunochemistry as follows: luminal A (ER-positive and/or PR-positive, HER2negative, and low histological grade [grade 1 or 2]); luminal B1 (ER-positive and/or PR-positive, and HER2-positive); luminal B2 (ER-positive and/or PR-positive, HER2-negative, and high histological grade [grade 3]), HER2-positive (ER-negative, PRnegative, and HER2-positive); and triple-negative (ER-negative, PR-negative, and HER2-negative). ${ }^{19}$

\section{Statistical analysis}

The primary outcome was disease-free survival (DFS), defined as the time interval from the date of surgery to the date of first recurrence, including local or distant. Survival curves were estimated using the Kaplan-Meier method, and survival differences were analyzed by log-rank test. The clinicopathological variables were analyzed in univariate and multivariate analyses of DFS with Cox proportional hazards model. Statistical analysis was performed using the R v3.5.1 (R Foundation for Statistical Computing, Vienna, Austria).

\section{RESULTS}

\section{Patient characteristics}

Patients were divided into two groups according to multiplicity. We found breast cancers involving a single mass in 4,744 cases $(82.4 \%)$ and breast cancers involving multiple masses in 1,014 cases (17.6\%). Table 1 shows the results of the comparison between patients with a single mass and patients with multifocal or multicentric masses.

Patients with multiple cancers were more likely to be young and have undergone total mastectomy. Statistically significant differences in lymph node positivity (single $38.0 \%$ vs multiple $47.3 \%, \mathrm{p}<.001$ ) and lymphatic invasion (single $24.7 \%$ vs multiple $32.6 \%, \mathrm{p}<.001$ ) were found between the two groups. In addition, multiplicity was associated with non-high histological grade $(\mathrm{p}<.001)$, ER positivity $(\mathrm{p}<.001)$, PR positivity $(\mathrm{p}<.001)$, and HER2 negativity $(\mathrm{p}=.003)$ of tumor. Therefore, breast cancers with multiple masses were more likely to have luminal A molecular subtype and less likely to be triple-negative subtype compared to those with a single mass $(\mathrm{p}<.001)$. 


\section{Disease-free survival}

DFS was evaluated in patients with single breast mass and multiple masses. Kaplan-Meier survival analysis indicated that

Table 1. Distribution of clinicopathological factors in single and multiple breast cancers $(n=5,758)$

\begin{tabular}{|c|c|c|c|}
\hline Characteristic & $\begin{array}{c}\text { Single } \\
(n=4,744)\end{array}$ & $\begin{array}{c}\text { Multiple } \\
(n=1,014)\end{array}$ & $\mathrm{p}$-value \\
\hline Age (yr) & & & .001 \\
\hline$<47$ & $2,459(51.8)$ & $591(58.3)$ & \\
\hline$\geq 47$ & $2,285(48.2)$ & $423(41.7)$ & \\
\hline Operation & & & $<.001$ \\
\hline Partial & $3,089(65.1)$ & $470(46.4)$ & \\
\hline Total & $1,655(34.9)$ & $544(53.6)$ & \\
\hline Chemotherapy & & & .115 \\
\hline Not done & $1,113(23.5)$ & $214(21.1)$ & \\
\hline Done & $3,631(76.5)$ & $800(78.9)$ & \\
\hline Hormonal therapy & & & $<.001$ \\
\hline Not done & $1,412(29.8)$ & $214(21.1)$ & \\
\hline Done & $3,332(70.2)$ & 800 (78.9) & \\
\hline Radiotherapy & & & $<.001$ \\
\hline Not done & $1,241(26.2)$ & $426(42.0)$ & \\
\hline Done & $3,503(73.8)$ & $588(58.0)$ & \\
\hline pT & & & .016 \\
\hline $\mathrm{T} 1$ & $2,720(57.3)$ & $618(60.9)$ & \\
\hline T2 & $1,827(38.5)$ & $370(36.5)$ & \\
\hline T3 & $197(4.2)$ & $26(2.6)$ & \\
\hline Lymph node & & & $<.001$ \\
\hline Negative & $2,941(62.0)$ & $534(52.7)$ & \\
\hline Positive & $1,803(38.0)$ & $480(47.3)$ & \\
\hline Anatomic stage group & & & .064 \\
\hline Stage I & $2,014(42.5)$ & $390(38.5)$ & \\
\hline Stage II & $2,089(44.0)$ & $479(47.2)$ & \\
\hline Stage III & $641(13.5)$ & $145(14.3)$ & \\
\hline Lymphatic invasion & & & $<.001$ \\
\hline Negative & $3,572(75.3)$ & $683(67.4)$ & \\
\hline Positive & $1,172(24.7)$ & $331(32.6)$ & \\
\hline Histology grade & & & $<.001$ \\
\hline Grade 1, 2 & $3,089(65.1)$ & $737(72.7)$ & \\
\hline Grade 3 & $1,655(34.9)$ & $277(27.3)$ & \\
\hline ER status & & & $<.001$ \\
\hline Negative & $1,430(30.1)$ & $232(22.9)$ & \\
\hline Positive & $3,314(69.9)$ & $782(77.1)$ & \\
\hline PR status & & & $<.001$ \\
\hline Negative & $1,789(37.7)$ & $278(27.4)$ & \\
\hline Positive & $2,955(62.3)$ & 736 (72.6) & \\
\hline HER2 status & & & .003 \\
\hline Negative & $3,448(72.7)$ & 783 (77.2) & \\
\hline Positive & $1,296(27.3)$ & $231(22.8)$ & \\
\hline Molecular subtype & & & $<.001$ \\
\hline Luminal A & $2,228(47.0)$ & $580(57.2)$ & \\
\hline Luminal B1 & $753(15.9)$ & 125 (12.3) & \\
\hline Luminal B2 & $410(8.6)$ & $94(9.3)$ & \\
\hline HER2 positive & $543(11.4)$ & $106(10.5)$ & \\
\hline Triple negative & $810(17.1)$ & $109(10.7)$ & \\
\hline
\end{tabular}

Values are presented as number (\%). patients with multiple masses had significantly poorer DFS than did those with a single mass ( 5 -year rate, $88.2 \%$ vs $85.2 \%$; $=$ .016) (Fig. 1A). When patients were subclassified according to T-category, Kaplan-Meier survival analysis in the T1 category group revealed significantly worse DFS for multiple breast cancer (5-year rate, $91.3 \%$ vs $87.4 \% ; \mathrm{p}=.033$ ) (Fig. 1B). There was no significant prognostic difference in $\mathrm{T} 2$ and $\mathrm{T} 3$ category groups $(\mathrm{p}=.093$ and $\mathrm{p}=.619$, respectively) (Fig. 1C, D). Using the anatomic stage group table in the AJCC eighth edition for tumor staging, breast cancer with multiplicity had poor prognosis in stage I ( 5 -year rate, $92.7 \%$ vs $90.3 \%$; $\mathrm{p}=.019)$ (Fig. $2 \mathrm{~A}$ ). When using the prognostic stage group table in the AJCC eighth edition, multiple breast masses were found to have significantly shorter DFS than single breast masses in stage group IA (5-year rate, $94.9 \%$ vs $88.7 \% ; \mathrm{p}=.032$ ) (Fig. 2B). However, no significant difference was found between single and multiple tumors in the other stage groups (i.e., anatomic staging group II or III and prognostic staging group IB, II, or III) (Fig. 2C).

Patients were divided into five molecular subtypes (i.e., luminal A, B1, and B2; HER2-positive; and triple-negative). The prognostic significance of multiplicity was only seen in patients with luminal A and HER2-positive groups in terms of DFS (5-year rate, $92.8 \%$ vs. $88.6 \% ; \mathrm{p}=.013$ and 5 -year rate, $86.9 \%$ vs $77.8 \%$; $\mathrm{p}=.003$, respectively) (Fig. 3A, B). There was no significant difference among the luminal B1 and B2 and triple-negative subtypes ( $\mathrm{p}=.937, \mathrm{p}=.453$, and $\mathrm{p}=.411$, respectively) (Fig. 3CE). In addition, when targeting patients with T1-2 N0 M0, hormone-receptor-positive, and HER2-negative cancer, Kaplan-Meier survival analysis revealed a significantly reduced DFS of multiple breast cancer (5-year rate, $95.2 \%$ vs $88.6 \%$; $\mathrm{p}=.031$ ) (Fig. 4).

Univariate analysis using Cox proportional hazard model indicated that high tumor stage (T3) (hazard ratio [HR], 2.44; 95\% confidence interval [CI], 1.84 to $3.23 ; \mathrm{p}<.001$ ), positive lymph node metastasis (HR, 2.06; 95\% CI, 1.8 to 2.36 ; $\mathrm{p}<.001$ ), high anatomic staging group (i.e., stage III) (HR, 3.47 ; $95 \%$ CI, 2.89 to 4.18; $<$ <.001), positive lymphatic emboli (HR, 2.16; 95\% CI, 1.88 to 2.49; $\mathrm{p}<.001$ ), high histological grade (i.e., grade 3) (HR, 1.52; 95\% CI, 1.33 to $1.74 ; \mathrm{p}<.001$ ), negative ER status (HR, 1.23; 95\% CI, 1.07 to $1.42 ; \mathrm{p}<.001$ ), positive HER2 status (HR, 1.21; 95\% CI, 1.05 to $1.40 ; \mathrm{p}=.004$ ), and the presence of multiplicity (HR, 1.24; 95\% CI, 1.04 to 1.48; $\mathrm{p}=.016)$ are significant variables associated with lower DFS (Table 2).

These significant factors in the univariate model were included in multivariate analysis, which demonstrated that tumor multiplicity correlated independently with worse DFS (adjusted HR, 

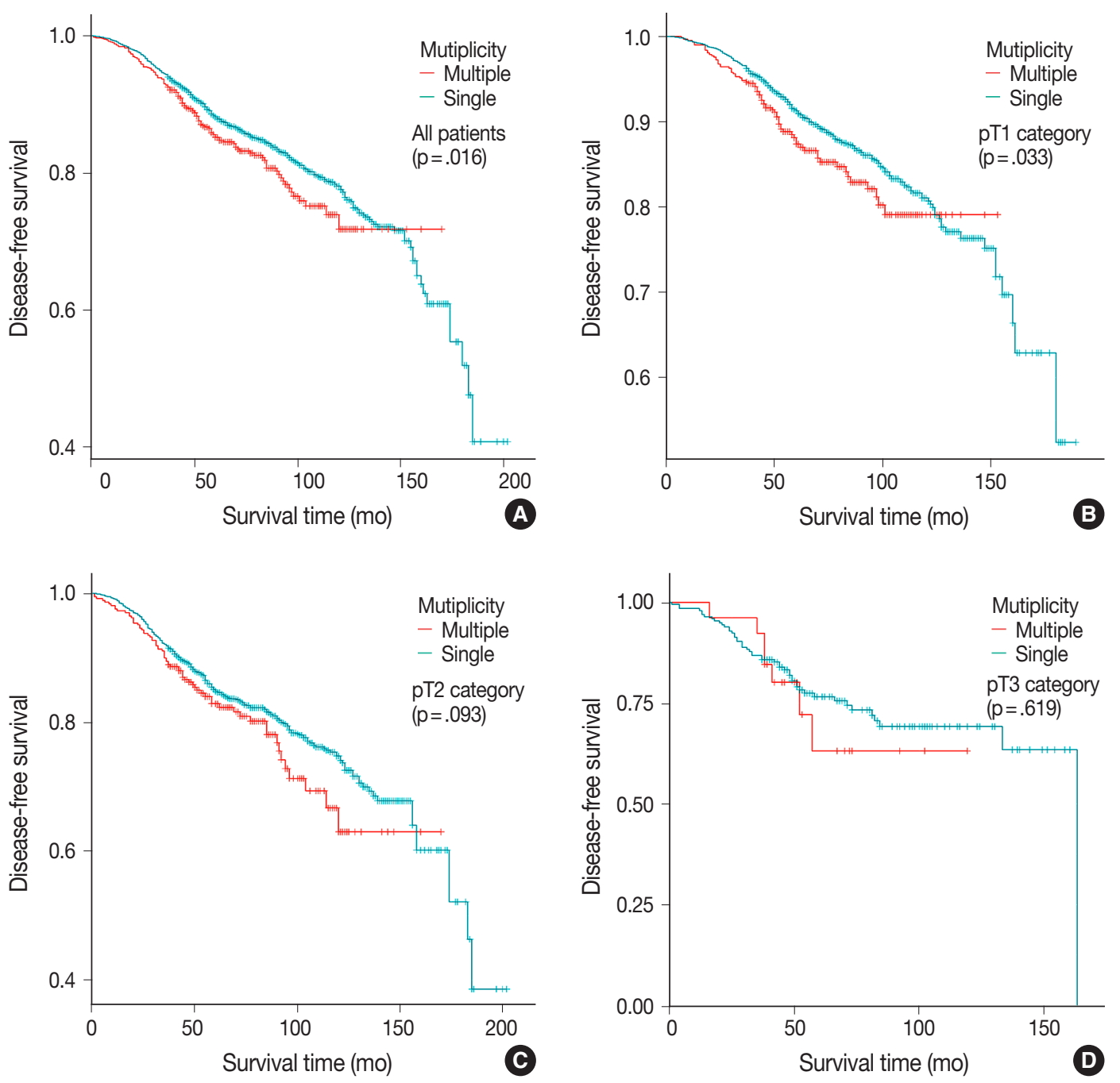

Fig. 1. The Kaplan-Meier curves for disease-free survival in patients with single and multiple masses according to T category. (A) Survival curve of all patients. (B) Survival curve of the T1 category. (C) Survival curve of the T2 category. (D) Survival curve of the T3 category. A significant difference is observed in the T1 category.

$1.23 ; 95 \% \mathrm{CI}, 1.05$ to $1.47 ; \mathrm{p}=.021)$. Other independent factors were high tumor stage (T3) (adjusted HR, 1.81; 95\% CI, 1.35 to $2.41 ; \mathrm{p}<.001$ ), positive lymph node metastasis (adjusted HR, 1.84; $95 \%$ CI, 1.60 to 2.13; $\mathrm{p}<.001$ ), and high histological grade (i.e., grade 3) (adjusted HR, 1.33; 95\% CI, 1.14 to 1.55; p < .001) (Table 3).

\section{DISCUSSION}

In the present study, the $17.6 \%$ incidence of surgically removed breast cancer with multiplicity is in line with prior data series. ${ }^{9,11,20-23}$ In previous studies, the incidence of multiple breast cancer had a wide range due to different definitions and inclusion criteria for multiple masses. Here, we used the term multiplicity if the cancer showed either multicentricity or multi- focality. Many researchers have studied the characteristics of multicentric or multifocal breast cancer. In the literature, lymphovascular invasion and axillary nodal involvement were more frequent in multicentric or multifocal breast cancers. ${ }^{3,5-15}$ The higher frequency of lymph node metastases could be due to the greater volume and surface area of multiple breast cancer or different biological behavior. ${ }^{8}$ In agreement with reported series, patients in this study with multiple masses had a higher incidence of lymph node involvement than patients with single mass. In addition, multiplicity was associated with frequent lymphovascular invasion.

Theoretically, as breast cancers with multiplicity are more likely to have lymph node involvement and lymphovascular invasion, it could be inferred that prognosis would be worse than that of single mass breast cancers. Of course, many researchers have studied multiplicity as a prognostic factor in breast cancer. However, 

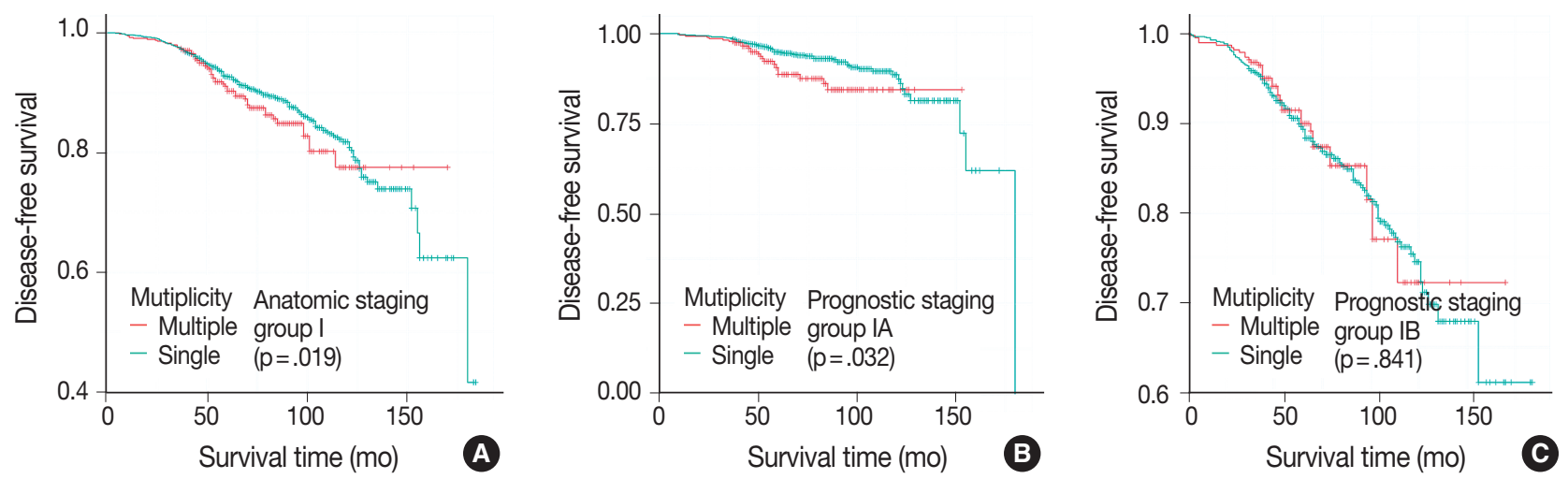

Fig. 2. The Kaplan-Meier survival curves for disease-free survival of patients with single and multiple masses in different Anatomic and prognostic staging groups. (A) Survival curve of the anatomic staging group I. (B) Survival curve of the prognostic staging group IA. (C) Survival curve of the prognostic staging group IB. The anatomic staging group I and prognostic staging group IA show a significant difference.
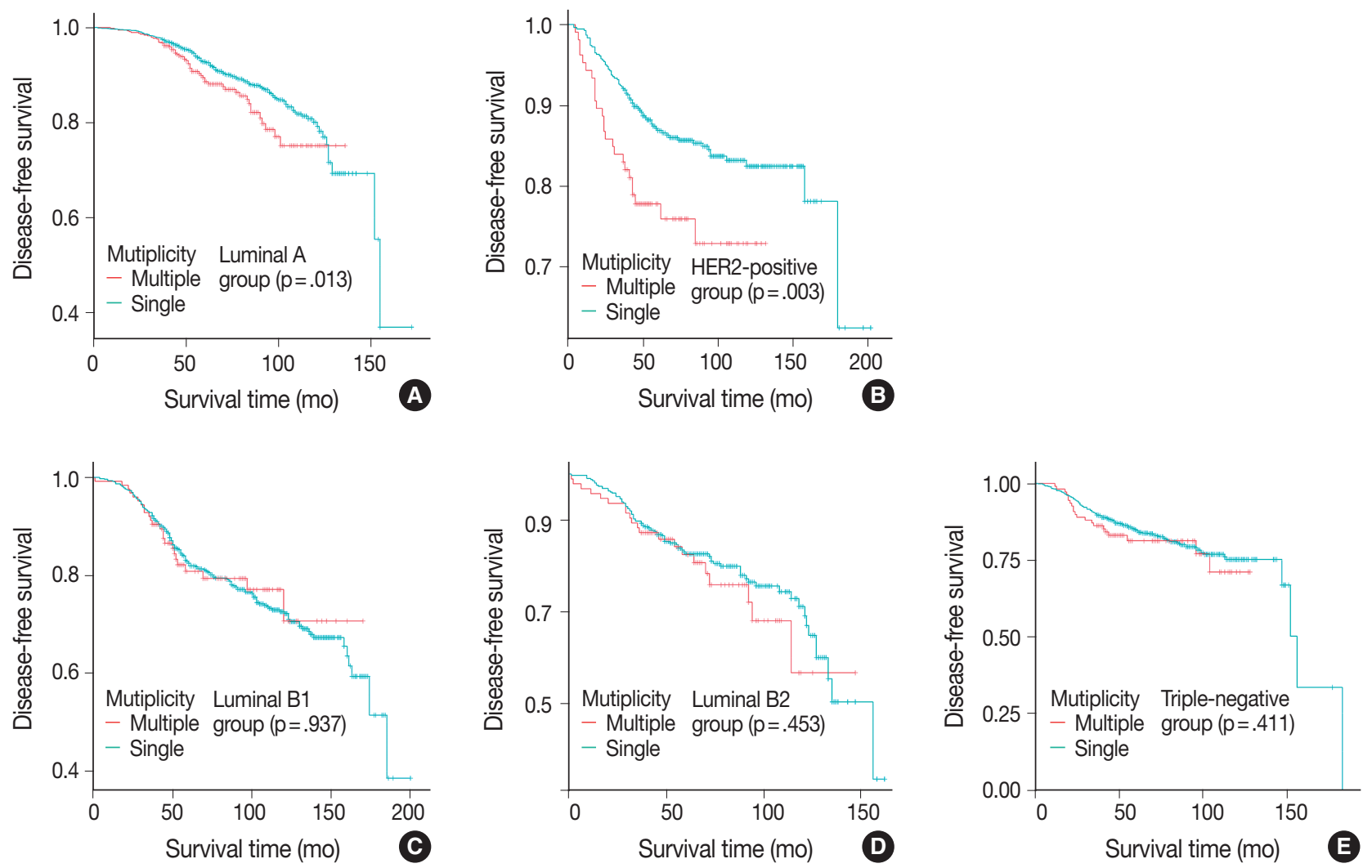

Fig. 3. Molecular subgroup analysis of the association between multiplicity and disease-free survival. The Kaplan-Meier survival curve of luminal A (A), human epidermal growth factor receptor 2 (HER2)-positive (B), luminal B1 (C), luminal B2 (D), and triple-negative groups (E). The difference is significant in patients of the luminal A and HER2-positive groups.

the biological and clinical significances of multiplicity are still debated. ${ }^{37,9,11,12,14,20,21,24-26}$ Vlastos et al. ${ }^{11}$ studied 284 patients with early-stage breast cancer and found that locoregional recurrence, distant metastasis, and disease-specific survival and DFS were not different between multicentric versus unicentric tumors. On the other hand, Yerushalmi et al. ${ }^{3}$ analyzed 1,554 patients and found multicentric/multifocal tumors to be associated with worse breast cancer-specific survival. Additionally, Neri et al. ${ }^{22}$ reported on 191 cases of breast cancer and found multifocal/multicentric breast cancer to be related to significantly worse prognosis with breast cancer-specific survival.

The results of our study suggest that multicentric and multi- 


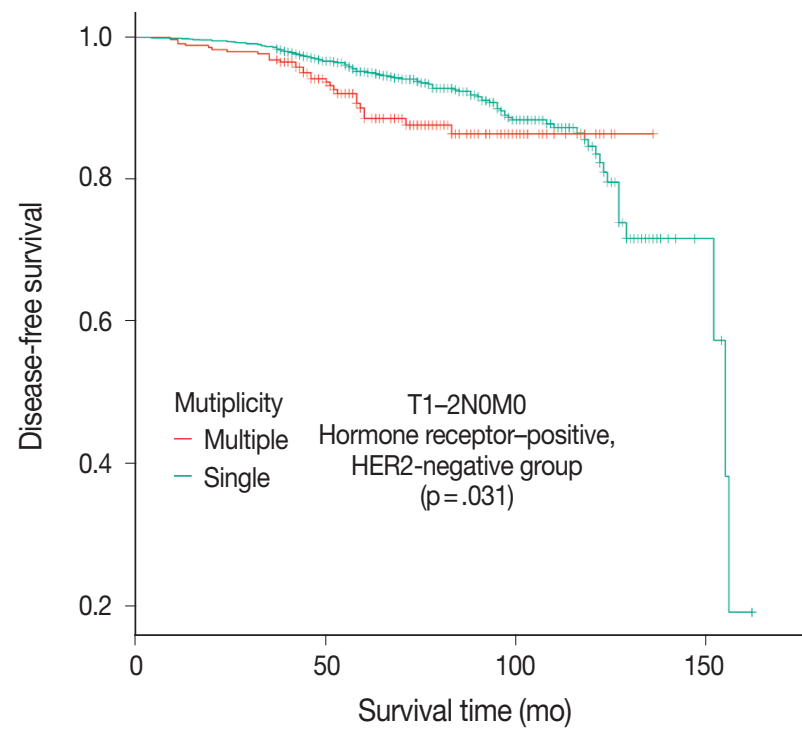

Fig. 4. The Kaplan-Meier survival curves for disease-free survival in patients with T1-2 NO M0, hormone receptor-positive, and human epidermal growth factor receptor 2 (HER2)-negative cancer. Comparison between patients with single mass and multiple masses.

focal breast cancers may have different biological behaviors. Multiple masses were more likely to have non-high histology grade, ER positivity, PR positivity, and HER2 negativity compared with single mass cases. Interestingly, we found that breast cancers with multiplicity were associated with luminal A molecular subtype and non-high histology grade, which are known to have good prognosis. Additionally, multiple breast masses of the luminal A group were found to have a significantly shorter DFS than single breast masses in Kaplan-Meier survival analysis ( $\mathrm{p}=.013)$. As with luminal A, multiplicity had prognostic significance in the HER2-positive group. According to our results, close observation during follow-up is needed, especially in patients of the luminal $A$ and HER2-positive groups with multiple breast cancer. There have been conflicting reports about hormonal receptor status. ${ }^{22,27}$ As in our study, Moon $e t$ al. ${ }^{27}$ identified frequent ER positivity and HER2 negativity of multiple breast cancers in a series of 2,882 patients. Conversely, however, Neri et al..$^{22}$ reviewed 1,158 patients and found an association between multiplicity and ERnegative and HER2-positive status. On the other hand, Moon et al..$^{27}$ reported that the difference in overall survival was significant only in patients with the triple-negative subtype.

Our results show that breast cancer with multiplicity has a negative effect on DFS, especially in early-stage cancer. The results of multivariate analysis confirmed the independent prognostic value of multiplicity, and Kaplan-Meier survival curve showed significantly reduced DFS for patients with multiple masses in the T1 stage group ( $\mathrm{p}=.033)$. The AJCC eighth edition
Table 2. Univariate Cox proportional hazards ratio analysis

\begin{tabular}{|c|c|c|c|}
\hline & Hazard ratio & $95 \% \mathrm{Cl}$ & $p$-value \\
\hline \multicolumn{4}{|l|}{ Multiplicity } \\
\hline Single & 1 & & \\
\hline Multiple & 1.24 & $1.04-1.48$ & .016 \\
\hline \multicolumn{4}{|l|}{ Age (yr) } \\
\hline$\geq 47$ & 1 & & \\
\hline$<47$ & 1.08 & $0.94-1.24$ & .258 \\
\hline \multicolumn{4}{|l|}{ pT } \\
\hline $\mathrm{T} 1$ & 1 & & \\
\hline $\mathrm{T} 2$ & 1.57 & $1.37-1.81$ & $<.001$ \\
\hline T3 & 2.44 & $1.84-3.23$ & $<.001$ \\
\hline \multicolumn{4}{|l|}{ Lymph node } \\
\hline Negative & 1 & & \\
\hline Positive & 2.06 & $1.80-2.36$ & $<.001$ \\
\hline \multicolumn{4}{|l|}{ Anatomic stage } \\
\hline Stage I & 1 & & \\
\hline Stage II & 1.65 & $1.40-1.95$ & $<.001$ \\
\hline Stage III & 3.47 & $2.89-4.18$ & $<.001$ \\
\hline \multicolumn{4}{|c|}{ Lymphatic invasion } \\
\hline Negative & 1 & & \\
\hline Positive & 2.16 & $1.88-2.49$ & $<.001$ \\
\hline \multicolumn{4}{|l|}{ Histology grade } \\
\hline Grade 1, 2 & 1 & & \\
\hline Grade 3 & 1.52 & $1.33-1.74$ & $<.001$ \\
\hline \multicolumn{4}{|l|}{ ER status } \\
\hline Positive & 1 & & \\
\hline Negative & 1.23 & $1.07-1.42$ & .004 \\
\hline \multicolumn{4}{|l|}{ HER2 status } \\
\hline Negative & 1 & & \\
\hline Positive & 1.21 & $1.05-1.40$ & .009 \\
\hline \multicolumn{4}{|c|}{ Molecular Subtype } \\
\hline Luminal A & 1 & & \\
\hline Luminal B1 & 1.8 & $1.49-2.18$ & $<.001$ \\
\hline Luminal B2 & 2.17 & $1.73-2.71$ & $<.001$ \\
\hline HER2 positive & 1.37 & $1.09-1.73$ & .007 \\
\hline Triple negative & 1.83 & $1.51-2.23$ & $<.001$ \\
\hline
\end{tabular}

$\mathrm{Cl}$, confidence interval; ER, estrogen receptor; HER2, human epidermal growth factor receptor 2 .

presents the Prognostic Stage Group table in addition to the anatomic stage group table using the T, N, and $\mathrm{M}$ categories. The Prognostic Stage Group table includes the anatomical T, N, and $\mathrm{M}$ categories; tumor grade; and the status of $\mathrm{ER}, \mathrm{PR}$, and HER2 biomarkers. The prognostic significance of multiplicity in terms of DFS was only seen in patients with anatomic staging group I and prognostic staging group IA by Kaplan-Meier survival analysis ( $\mathrm{p}=.019$ and $\mathrm{p}=.032$, respectively). Therefore, the negative prognostic impact of multiplicity could be considered for subclassification in at least early breast cancer patients.

The Oncotype Dx genomic test is now performed for consideration of adjuvant chemotherapy in patients with T1-2 N0 M0, hormone receptor-positive, and HER2-negative cancer. ${ }^{28}$ 
Table 3. Multivariate Cox proportional hazards ratio analysis

\begin{tabular}{lllc}
\hline & Hazard ratio & $95 \% \mathrm{Cl}$ & $\mathrm{p}$-value \\
\hline Multiplicity & & & \\
Single & 1 & & \\
Multiple & 1.23 & $1.05-1.47$ & .021 \\
PT & & & \\
T1 & 1 & & .002 \\
T2 & 1.27 & $1.09-1.47$ & \\
T3 & 1.81 & $1.35-2.41$ & $<.001$ \\
Lymph node & & & \\
Negative & 1 & & \\
Positive & 1.84 & $1.60-2.13$ & $<.001$ \\
Histology grade & & & \\
Grade 1, & 1 & & \\
Grade 3 & 1.33 & $1.14-1.55$ & $<.001$ \\
ER status & & & \\
Positive & 1 & & \\
Negative & 1.09 & $0.93-1.28$ & .255 \\
HER2 status & & & \\
Negative & 1 & & \\
Positive & 1.1 & $0.95-1.28$ & .206 \\
\hline
\end{tabular}

$\mathrm{Cl}$, confidence interval; ER, estrogen receptor; HER2, human epidermal growth factor receptor 2 .

In this patient population in our study, multiple breast masses were found to have a significantly shorter DFS than single breast mass $(\mathrm{p}=.031)$. Based on the difference of prognosis, adjuvant chemotherapy would be necessary for multiple breast masses even without the Oncotype Dx test.

Our study has several limitations. First, this retrospective study had a relatively short-term follow-up period (median duration, 64 months). Second, molecular subtype was evaluated only using the largest among multiple masses. Because intertumoral heterogeneity could be a factor affecting survival, a further study should be conducted to investigate the relationship between intertumoral heterogeneity and survival in multiple breast cancer. Finally, patients with neoadjuvant therapy were not included. Therefore, the evaluation of advanced stage breast cancer was relatively limited.

In conclusion, the results of this study indicate that tumor multiplicity is frequently found in luminal A breast cancer, is associated with frequent lymph node metastasis, and is correlated with worse DFS. Tumor multiplicity has prognostic value and could be used to subclassify invasive breast cancer in the early stage. Adjuvant chemotherapy would be necessary for multiple breast masses of the T1-2 N0 M0, hormone-receptorpositive, and HER2-negative cancer groups.

\section{ORCID}

Jinah Chu: https://orcid.org/0000-0001-9050-084X

Hyunsik Bae: https://orcid.org/0000-0002-5347-466X

Youjeong Seo: https://orcid.org/0000-0003-4595-3836

Soo Youn Cho: https://orcid.org/0000-0001-9714-7575

Seok-Hyung Kim: https://orcid.org/0000-0001-6298-5958

Eun Yoon Cho: https://orcid.org/0000-0001-5849-3600

\section{Conflicts of Interest}

No potential conflict of interest relevant to this article was reported.

\section{REFERENCES}

1. Qualheim RE, Gall EA. Breast carcinoma with multiple sites of origin. Cancer 1957; 10: 460-8.

2. Bendifallah S, Werkoff G, Borie-Moutafoff C, et al. Multiple synchronous (multifocal and multicentric) breast cancer: clinical implications. Surg Oncol 2010; 19: e115-23.

3. Yerushalmi R, Kennecke H, Woods R, Olivotto IA, Speers C, Gelmon KA. Does multicentric/multifocal breast cancer differ from unifocal breast cancer? An analysis of survival and contralateral breast cancer incidence. Breast Cancer Res Treat 2009; 117: 365-70.

4. Egan RL. Multicentric breast carcinomas: clinical-radiographicpathologic whole organ studies and 10-year survival. Cancer 1982; 49: 1123-30.

5. Tot T. Axillary lymph node status in unifocal, multifocal, and diffuse breast carcinomas: differences are related to macrometastatic disease. Ann Surg Oncol 2012; 19: 3395-401.

6. Duraker N, Caynak ZC. Axillary lymph node status and prognosis in multifocal and multicentric breast carcinoma. Breast J 2014; 20: $61-8$.

7. Cabioglu N, Ozmen V, Kaya H, et al. Increased lymph node positivity in multifocal and multicentric breast cancer. J Am Coll Surg 2009; 208: 67-74.

8. Andea AA, Bouwman D, Wallis T, Visscher DW. Correlation of tumor volume and surface area with lymph node status in patients with multifocal/multicentric breast carcinoma. Cancer 2004; 100: 20-7.

9. Joergensen LE, Gunnarsdottir KA, Lanng C, Moeller S, Rasmussen BB. Multifocality as a prognostic factor in breast cancer patients registered in Danish Breast Cancer Cooperative Group (DBCG) 1996-2001. Breast 2008; 17: 587-91.

10. Coombs NJ, Boyages J. Multifocal and multicentric breast cancer: does each focus matter? J Clin Oncol 2005; 23: 7497-502. 
11. Vlastos G, Rubio IT, Mirza NQ, et al. Impact of multicentricity on clinical outcome in patients with T1-2, N0-1, M0 breast cancer. Ann Surg Oncol 2000; 7: 581-7.

12. Rezo A, Dahlstrom J, Shadbolt B, et al. Tumor size and survival in multicentric and multifocal breast cancer. Breast 2011; 20: 259-63.

13. Lynch SP, Lei $X$, Chavez-MacGregor M, et al. Multifocality and multicentricity in breast cancer and survival outcomes. Ann Oncol 2012; 23: 3063-9.

14. Pedersen L, Gunnarsdottir KA, Rasmussen BB, Moeller S, Lanng C. The prognostic influence of multifocality in breast cancer patients. Breast 2004; 13: 188-93.

15. Tot T, Gere M, Pekár G, et al. Breast cancer multifocality, disease extent, and survival. Hum Pathol 2011; 42: 1761-9.

16. Giuliano AE, Edge SB, Hortobagyi GN. Eighth edition of the AJCC cancer staging manual: breast cancer. Ann Surg Oncol 2018; 25: 1783-5.

17. Hammond ME, Hayes DF, Dowsett M, et al. American Society of Clinical Oncology/College Of American Pathologists guideline recommendations for immunohistochemical testing of estrogen and progesterone receptors in breast cancer. J Clin Oncol 2010; 28: 2784-95.

18. Wolff AC, Hammond ME, Hicks DG, et al. Recommendations for human epidermal growth factor receptor 2 testing in breast cancer: American Society of Clinical Oncology/College of American Pathologists clinical practice guideline update. Arch Pathol Lab Med 2014; 138: 241-56.

19. Goldhirsch A, Wood WC, Coates AS, et al. Strategies for subtypes: dealing with the diversity of breast cancer: highlights of the St. Gallen
International Expert Consensus on the Primary Therapy of Early Breast Cancer 2011. Ann Oncol 2011; 22: 1736-47.

20. Litton JK, Eralp Y, Gonzalez-Angulo AM, et al. Multifocal breast cancer in women $<$ or $=35$ years old. Cancer 2007; 110: 1445-50.

21. Boyages J, Jayasinghe UW, Coombs N. Multifocal breast cancer and survival: each focus does matter particularly for larger tumours. Eur J Cancer 2010; 46: 1990-6.

22. Neri A, Marrelli D, Megha T, et al. “Clinical significance of multifocal and multicentric breast cancers and choice of surgical treatment: a retrospective study on a series of 1158 cases". BMC Surg 2015; 15: 1.

23. O'Daly BJ, Sweeney KJ, Ridgway PF, et al. The accuracy of combined versus largest diameter in staging multifocal breast cancer. J Am Coll Surg 2007; 204: 282-5.

24. Weissenbacher TM, Zschage M, Janni W, et al. Multicentric and multifocal versus unifocal breast cancer: is the tumor-node-metastasis classification justified? Breast Cancer Res Treat 2010; 122: 27-34.

25. Ustaalioglu BO, Bilici A, Kefeli U, et al. The importance of multifo$\mathrm{cal} /$ multicentric tumor on the disease-free survival of breast cancer patients: single center experience. Am J Clin Oncol 2012; 35: 580-6.

26. Fish EB, Chapman JA, Link MA. Assessment of tumor size for multifocal primary breast cancer. Ann Surg Oncol 1998; 5: 442-6.

27. Moon HG, Han W, Kim JY, et al. Effect of multiple invasive foci on breast cancer outcomes according to the molecular subtypes: a report from the Korean Breast Cancer Society. Ann Oncol 2013; 24: 2298-304.

28. Sparano JA, Gray RJ, Makower DF, et al. Prospective validation of a 21-gene expression assay in breast cancer. N Engl J Med 2015; 373: 2005-14. 